Euskal ikerketen aldizkaria | Revue d'études basques |

Revista de estudios vascos | Basque studies review

$7 \mid 2002$

Numéro VII

\title{
Pierre Bidart, La singularité basque, PUF, 2001
}

\section{Manex Goyhenetche}

\section{OpenEdition}

\section{Journals}

Édition électronique

URL : http://journals.openedition.org/lapurdum/1064

DOI : 10.4000/lapurdum.1064

ISSN : 1965-0655

Éditeur

IKER

Édition imprimée

Date de publication : 1 octobre 2002

Pagination : 361-367

ISBN : 2-86781-321-2

ISSN : $1273-3830$

\section{Référence électronique}

Manex Goyhenetche, «Pierre Bidart, La singularité basque, PUF, 2001 », Lapurdum [En ligne], 7| 2002, mis en ligne le 01 juin 2009, consulté le 21 septembre 2020. URL : http://journals.openedition.org/ lapurdum/1064; DOI : https://doi.org/10.4000/lapurdum.1064 


\section{Comptes rendus - Liburu ikertzeak}

\section{Pierre Bidart, La singularité basque, PUF, 2001.}

C'est avec un très grand intérêt que j'ai lu le dernier ouvrage de Pierre Bidart ayant pour titre $L a$ singularité basque. Professeur des universités, enseignant au département d'anthropologie de Bordeaux II, ancien membre de la Casa Velazquez, P. Bidart (désormais P.B.), connu aussi pour ses nombreuses publications, a atteint une grande réputation.

Le dernier travail qu'il vient de faire paraître est d'envergure, dans une perspective anthropologique qui se veut globale et totale. Il embrasse l'ensemble des territoires du Pays Basque de la Biscaye à la Soule, situant l'analyse dans un temps long qui s'étend de l'époque médiévale (avec le Guide du Pélerin) jusqu'à nos jours y compris le temps immédiat du rock. C'est dire combien un tel ouvrage mérite lecture attentive, examen et notes critiques, exercice auquel je me suis volontiers livré, ma contribution se situant essentiellement dans le domaine de l'histoire.

En introduction, l'auteur commence par fixer le cadre de sa recherche qui a pour objet d'étudier "la production discursive" sur les Basques ou concernant le domaine basque. Il aborde d'emblée la question des conditions d'élaboration d'un tel discours qui constitue l'axe central de son travail.

Ensuite, P. B. passe rapidement en revue, en une sorte de synthèse introductive, les discours élaborés par des auteurs aussi divers que Frédéric Le Play (XIX ${ }^{e} \mathrm{~s}$.), G. Humboldt (XIX ${ }^{\mathrm{e}}$ s.) ou Aimeric Picaud (auteur du Codex ou Guide du Pélerin rédigé au XII ${ }^{\mathrm{e}} \mathrm{s}$ ). Tout cela sera explicité et développé dans le corps de l'ouvrage. Mais déjà une question se pose : pourquoi une étude approfondie de seulement ces auteurs (Frédéric Le Play ou Humboldt par exemple), tout en laissant de côté ou survolant très superficiellement les producteurs du XVIIIe siècle comme Rousseau, Voltaire et les Encyclopédistes qui ont aussi écrit abondamment sur les Basques? On aurait aimé savoir en quoi a consisté l'apport du siècle des Lumières à l'élaboration du discours (car ils en ont un) et quelle en était la singularité. Voilà un passionnant sujet d'étude qui concerne les décennies précédant la Révolution française.

P. B. évoque le chêne de Gernika considéré comme une "formule métaphorique", "au sein du récit fondateur de la "nation" basque". En fait, l'auteur ne développera pas dans le corps de l'ouvrage cet aspect important, se contentant, à notre avis, d'utiliser un lieu commun, sans renouveler son approche et son contenu. Car si on veut insérer le Chêne de Gernika dans la production discursive (pourquoi pas ?), encore faut-il traiter la question sous tous. ses aspects. Tout d'abord, il faut commencer par se demander ce qu'a été le Chêne de Gernika avant le XIX ${ }^{\mathfrak{e}}$ siècle. L'interrogation nous renvoie au for de Biscaye de 1453 (Fuero Viejo), dont les articles 13 à 20, 50, 36 et 52,61, 63 assurent, de manière bien plus explicite que l'Habeas Corpus, la sauvegarde des libertés individuelles "sos el arbol de Guemica". Nous avons là tout une production discursive élaborée dès la fin du Moyen Age par la noblesse et la bourgeoisie biscayennes (à l'instar d'ailleurs d'autres sociétés 
maritimes, comme la République de Venise et les Provinces-Unies).

On peut encore pousser la réflexion plus en profondeur. Iparraguirre a créé Gernikako Arbola en 1853, cinq ans après la Révolution de 1848, la même année où G. Sorrieu composa son célèbre tableau sur le rêve de la République universelle qui anima les peuples de l'Europe au milieu du XIX ${ }^{\mathrm{e}}$ siècle, dans le sillage de l'héritage de la Révolution française. Rappelons qu'lparraguirre fut emprisonné à Paris pour avoir chanté $L a$ Marseillaise. Cela mérite réflexion et analyse. De G. Sorrieu à Iparraguirre, nous trouvons la même philosophie de la liberté.

Toujours à la p. 14, P. B. évoque "la très grande récurrence Eskualdun fededun (...) comme norme idéologique évidente pour tout Basque". Certes, à lire la littérature de l'Eglise catholique du dernier tiers du XIX siècle, le "vrai" Basque est identifié au Basque croyant, selon un archétype qui traverse bien des pensées et des religions de par le monde. Ce n'est pas propre à la "production discursive" basque, d'autant que ce n'est qu'un aspect de la question. Car pendant cette même période du XIX siècle il y a eu l'autre littérature, celle des "rouges" (pour reprendre une expression classique de la littérature basque), héritiers de la Révolution française, bascophiles regroupés autour du Réveil basque. Et ceci ne fait qu'illustrer les faiblesses épistémologiques de l'ouvrage. Car comment peut-on étudier la "production discursive" "Eskualdun Fededun" (en citant par exemple six fois l'hebdomadaire Eskualduna qui véhicula le concept, et ne faire aucune mention du Réveil basque organe de la "production discursive" des républicains bascophiles?

Et si on quitte le dernier tiers du XIX ${ }^{\mathfrak{e}}$ siècle (qu'il faut d'ailleurs situer dans son contexte historique), pour faire un retour sur le XVIII" siècle (celui de "l'Etat-nation Ilustrado") (p. 15), la documentation existante met bien en évidence l'impact du mouvement encyclopédiste en Pays Basque, qu'il s'agisse des frères Garat, d'Ustaritz à Bordeaux, à Versailles et à Paris, ou des activités de la Real Sociedad Bascongada de Los Amigos del Pais (complètement passée sous silence dans le travail de P.B.) dont le rôle fut déterminant dans l'évolution politique, culturelle, sociale et économique du Pays Basque. Il y a une production discursive de la Real Sociedad Bascongada du XVIII ${ }^{\mathbf{e}}$ siècle, d'inspiration moderne, basque et universaliste, dont une partie demanda en 1794 la formation d'une "République indépendante comme avant 1200" et son rattachement à la France. Les événements de la guerre de Convention suscitèrent au moins trois sortes de "production discursive" (Moncey, Tallien, Cavaignac).

De plus, les pages 16 et 17 contiennent un raccourci très schématique (trop schématique) dans la mesure où l'auteur prétend que "la pensée religieuse et cléricale du XIX ${ }^{e}$ siècle" (en opposition et en confrontation avec la Révolution française), "la littérature religieuse et cléricale du XIX ${ }^{e}$ siècle au XX $X^{e}$ siècle" sont à la source de la "vision que le discours nationaliste basque reprendra dans la seconde moitié de ce siècle entièrement à son compte". Pour peu qu'on veuille se livrer à un véritable examen du matériau documentaire, on est obligé de reconnaitre que le nationalisme basque, dans la seconde moitié du XX $\mathrm{XX}^{\varepsilon}$ siècle, s'est abreuvé à des sources autres que celle de l'Eglise catholique; mentionnons rapidement les luttes de décolonisation, les guerres de libération nationale (Algérie, Viet Nam), les combats anti-impérialistes, les modèles de développement économique inspirés du coopérativisme (influence du modèle yougoslave par exemple), sans oublier les différents apports marxistes d'expression bascophone. De tout cela, aucune mention n'est 
faite dans le travail de P.B. ; c'est dommage, dans la mesure où il prétend embrasser aussi le $\mathrm{XX}^{\mathrm{e}}$ siècle.

La première partie de l'ouvrage est consacrée à la "graphie des singularités basques" (p. 19-68). P.B. commence par examiner "le discours négatif" sur les Basques (p. 20) qu'il fait remonter au Codex, déjà cité. Mais "la légende noire des Basques" commence avec les auteurs romains (Salluste, Valère-Maxime, Juvénal); elle continue avec les auteurs chrétiens de l'Antiquité tardive et du haut Moyen Age (Ausone, Paulin), les chroniqueurs francs comme Grégoire de Tours ou Eginhard. Dans cette perspective, "la singularisation négative des Basques" ne date pas du XVIII" siècle.

Dans ce survol historique, Oihenart est à peine évoqué. Or, il y avait là un intéressant développement à faire, car c'est bien Oihenart qui a détruit la production discursive mythique héritée du Moyen Age et que reprit Larramendi au XVIII ${ }^{\mathrm{e}}$ siècle (celui-ci est amplement étudié et à juste titre). Et Oihenart, à son tour, a élaboré une production discursive spécifique, insérant déjà les traits de caractère ethnologique ou linguistique du Pays Basque, dans le sillage de l'héritage de la Renaissance et de l'Humanisme.

En ce qui concerne "la singularité positive", P.B. reprend les thèses désormais classiques sur Larramendi, Moguel, Herder, Humboldt, tout cela débouchant à la fin du $\mathrm{XIX}^{\mathrm{e}}$ siècle, d'après l'auteur, sur la pensée et l'œuvre de Sabino Arana "pour justifier un projet politique d'épuration ethnique". Soit, mais on peut dire la même chose de toute l'Europe au XIX siècle et notamment de la France; n'oublions pas la littérature d'expression française et sur les Français, de Gobineau à $H$. Chavée, théoriciens du racisme français à la même époque. Dans ce contexte, qu'a de spécial la production discursive basque? Rien. Elle partage les modes de pensée en vogue à l'époque.

P.B. établit ensuite un parallèle (une "homologie structurelle") (p. 29-39) entre la devise "Eskualdun fededun" (XIX siècle) et "Eskuaraz Bizi" (XX ${ }^{\mathfrak{e}}$ siècle). Cela aussi constitue un raccourci trop schématique. Où est la "singularité basque" lorsque dans toutes les églises de France et de Navarre on chantait "Catholiques et Français toujours"? P. B. ne nous donne aucun matériau documentaire, passe sous silence des questions d'ordre méthodologique. Autour de quel projet par exemple est née la devise "Euskaraz Bizi"? Quel est son contenu réel ? Surtout, la critique d" "Euskaraz. Bizi", en dehors de l'énoncé critique, n'aborde aucune analyse.

Prolonger la "modélisation ethnico-religieuse" jusqu'à la naissance en 1969 du mouvement Fededunak laisse perplexe en ce qui concerne la méthodologie et le traitement documentaire. L'approche des questions touchant les pratiques et les comportements religieux ne dispense pas des règles méthodologiques de sociologie et d'anthropologie, de la nécessité de situer toute prémisse dans le contexte historique qui lui est dû. Or, en ce qui concerne le mouvement Fededunak, parler de "modélisation ethnico-religieuse" constitue sans doute une formule ramassée, frappante, consacrée par le vocabulaire médiatique en vogue, mais ne répond guère aux critères d'épistémologie que l'anthropologue aussi se doit de respecter. Où est le matériau documentaire? où est l'historique de l'Eglise catholique en Pays Basque ? où sont étudiées les conditions et le contexte d'élaboration qui contribuèrent à la naissance de Fededunak ? Pour répondre à ces questions, au moins trois pistes d'exploration s'offrent au chercheur: 1. D'une manière générale, l'histoire de l'Eglise elle- 
même en Pays Basque, 2. L'évolution de la liturgie au moins depuis la fin de la Seconde Guerre mondiale, 3. Les modalités de passage d'une liturgie et d'une catéchèse d'expression basque vers une pratique quasi exclusive du français dans certaines paroisses (rôle du clergé ? rôle des laïcs? pression des nouveau-arrivés?).

P. B. se livre ensuite à l'examen des "activités mythologiques et scientifiques" ( $p$. 39-40). Trois personnages sont mis en parallèle: A. Chaho, J.M. Barandiaran, J. Mirande. Voilà un procédé bien simplificateur. Pourquoi et au nom de quelle prémisse avoir choisi ces seuls trois auteurs qui couvrent une période de 150 ans? Pendant cette période longue, la "production discursive" s'est-elle cristallisée uniquement autour de ces trois auteurs ? Sans doute servent-ils la démonstration que veut établir P. B? Mais pour la même période, en ce qui concerne, par exemple le seul champ littéraire dont J. Mirande n'a en rien l'exclusivité et le monopole, l'auteur aurait pu analyser, entr'autres, la production discursive de Gabriel Aresti, le poète "rouge" de Bilbao, totalement passé sous silence par P. B., celle de Koldo Mitxelena, militant anti-franquiste devenu spécialiste de la linguistique grâce à ses études durant son séjour dans les geôles franquistes, ou celle de Pierre Laffite dont la présentation n'est plus à faire. Nous aurions eu là, -et nous avons-, trois autres aspects de la "production discursive" sur la "basquité". Peut-on faire le survol de cette période sans même mentionner la "production discursive" de Julio Urquijo, fondateur de la Revue Internationale des Etudes Basques, d'Eskualtzaleen Biltzarra, et qui joua un rôle de premier plan dans la renaissance de la culture et des études basques dans la première moitié du $\mathrm{XX}^{\mathrm{e}}$ siècle?

Comment parler de cette même période en laissant dans le silence Lizardi (Jose Maria de Aguirre) ? Outre ses oeuvres de poésie, il fut membre fondateur de la célèbre société Euskaltzaleak, écrivit dans la presse locale de très nombreux articles sur l'école, le théâtre, l'enfance, prit part à toutes les luttes politiques et littéraires de son époque, et presque toujours en basque.

Comment ne pas évoquer la figure et l'œuvre de Jean Etchepare ? Fils d'émigrés en Argentine, grand lecteur de Haeckel et Nietzsche, installé comme médecin aux Aldudes, puis à Cambo, président d'Eskualizaleen Biltzarra, il écrivit en basque régulièrement, entre 1902 et 1914, dans l'hebdomadaire Eskualduna de nombreux articles traitant de questions aussi diverses que les relations internationales, la vie politique, les problèmes financiers, la recherche scientifique. Il est l'auteur d'ouvrages majeurs comme Buruxkak et Beribilez.

P.B. prolonge son analyse en examinant "le glissement progressif de la basquité" "vers une formulation sensiblement politique", ce qu'il appelle d'ailleurs "l'avénement d'une basquité active", fruit, à son avis, de "la pensée cléricale" caractérisée par le refus de la République. Ici aussi, examinons le matériau documentaire dont nous disposons. La documentation existante nous renvoie-t-elle seulement à la pensée cléricale, même si celleci reste dominante et triomphante dans une période où elle l'est dans l'ensemble de la France, sinon de l'Europe (n'oublions pas le Congrès de Vienne) ? Que fait-on de la "basquité active" se situant en dehors de "la pensée cléricale" : A. Chaho (que pourtant l'auteur étudie), Lewy d'Abartiague (totalement passé sous silence), les acteurs labourdins, bas-nayarrais et souletins de la Révolution française ? Où est l'analyse de la production discursive de Dominique Garat (totalement ignoré par l'auteur) ? Outre ses nombreuses interventions à l'Assemblée nationale constituante rapportées par Le Moniteur universel et 
le Journal des Assemblées Nationales, mentionnons l'important discours qu'il prononça en 1784 devant l'Académie de Bordeaux et dont la deuxième partie a pour titre "sur les Basques" (5). Nous avons là un document de premier choix.. Mentionnons aussi son intervention en 1795 en tant que Président de l'administration municipale du canton d'Ustaritz pour nommer un instituteur bascophone car, selon lui, "dans les communes basques, un instituteur doit savoir très bien le Basque et le français autrement les Ecoliers pourraient oublier le Basque, sans apprendre le français".

Certes, P. B. mentionne par trois fois Joseph Garat. Mais en dehors d'une évocation générale, il n'y a aucune analyse de sa production discursive qui a été fort importante, ne seraient-ce que ses projets de "Nouvelle Phénicie" (passés sous silence). Joseph Garat a rédigé des articles sur le Pays Basque et sur la langue basque dans l'Encyclopédie. Toujours en ce qui concerne les "frères Garat" (p. 54), on pourrait analyser un autre excellent matériau documentaire constituant à lui seul une "production discursive" spécifique: le cahier de doléances du Biltzar de Labourd rédigé en basque et en français.

Dans le même ordre d'idées, puisque la Révolution française reste une référence importante dans tout ce travail sur La singularité basque, on ne peut pas se dispenser d'un minimum d'approche historique sur la décennie 1789-1799. Or, la documentation d'archives montre que les premiers événements de la Révolution française ont été accueillis avec enthousiasme par les habitants du Pays Basque. Mais une première grande désillusion fut provoquée par le refus de reconnaître un département des "trois provinces basques" qui était réclamé par tout le monde.

Bref, l'étude des différents aspects de la "production discursive" sur la "basquité" au siècle des Lumières reste à faire. Si P.B. ne le fait pas, c'est qu'il a fait dès le départ le choix d'un a priori idéologique et méthodologique : les Basques seraient ataviquement réactifs à l'impact de la modernité tout au long de leur histoire. Bref, la "Wishfull thinking" sert de soubassement à la méthodologie de P.B., dans la superposition continuelle de ses désirs et de la réalité.

La fin de la première partie est consacrée à "la singularité ambivalente" renvoyée par l'image du "Basque valeureux" et du "Basque rebelle" (p. 53 et suivantes). Pour illustrer la "vaillance" des Basques, est analysé un texte de 1885 sur l'émigration. Mais on ne peut pas réduire celle-ci uniquement à ce trait de caractère. Tout émigré, à travers le monde, n'est-il pas destiné à la vaillance pour survivre? Pour ce qui concerne "le Basque rebelle" (p. 57), nous avons là un lieu commun de la littérature sociale et historique. Les situations d'insoumission et de désertion à la conscription ne sont en rien spécifiques au Pays Basque. C'est un phénomène qui a affecté les populations des régions frontalières comme l'a montré J.F. Soulet. On est étonné d'ailleurs que cette thèse ne soit pas convoquée. On y trouve pourtant des données historiques et anthropologiques essentielles pour la compréhension $\mathrm{du}$ comportement des populations pyrénéennes et de la production discursive dont elles ont été l'objet.

Du "Basque rebelle", il est tentant de passer à la question de "l'intégration" des Basques à l'Etat ( $\mathrm{p} .63$ ), en posant une prémisse, théorique, dont la véracité historique reste à démontrer: "comment domestiquer ce qui dans la basquité se dérobe aux exigences conjointes de la République et de la modernité?". Il suffit de parcourir les nombreux 
documents conservés dans les archives pour constater que les Basques de 1789 avaient su faire la synthèse de leur "basquité" et de la République. En fait, dès 1789, l'élite du Pays Basque, celle des notables, est "intégrée" à l'Etat monarchique puis républicain. La Révolution constitue même pour elle un ascenseur social. Et on pourrait très bien étudier (le matériau documentaire existe) la "production discursive" des élites du Pays Basque en 1789, au sein du Biltzar de Labourd comme des Etats de Navarre, ainsi que le processus d'intégration qui les caractérise, repérable dans le temps bien avant le XIX ${ }^{\mathrm{e}}$ siècle).

La deuxième partie de l'ouvrage est consacrée à "L'Aufklarung et la basquité" (p. 69-112). Pour résumer les propos de P.B., disons que dans le sillage de Herder, Humboldt et autres auteurs allemands, le Pays Basque est en quelque sorte une invention de l'Allemagne. Mais, avec ce même procédé, on pourrait dégager d'autres conclusions similaires parallèles. Le Pays Basque peut être aussi, dans ces conditions, considéré comme une invention de Victor Hugo et des voyageurs romantiques pour le XIX ${ }^{\mathrm{e}}$ siècle, des encyclopédistes et des philosophes des Lumières pour le XVIII ${ }^{\mathrm{e}}$ siècle, d'Oihenart et de sa Notitia Utriusquae Vasconiae pour le XVII ${ }^{\mathrm{E}}$ siècle. Et pourquoi le Pays Basque n'est-il pas une invention de la pensée européenne du $\mathrm{XVI}^{\mathrm{e}}$ sièclc, avec Shakespeare, Sébastien Moreau, Navagero, Cristobal Weiditz, Venturino, François de Belleforest, J.A. de Thou, Scaliger?

Dans la troisième partie, P.B. revient sur A. Chaho, J.M. Barandiaran et Mirande dont il situe l'œuvre et l'influence dans le temps "entre mythologisme et positivisme" (p. 113-140). Dans la forêt de la littérature basque, pourquoi cette insistance quasi exclusive sur un arbre, celui du poète souletin Jon Mirande? Parce qu'il s'agit d'une représentant de "l'idéologie nationaliste fascisante" (p. 34, 39) ? Par ailleurs, prétendre que Jon Mirande est "à l'origine du renouvellement de la poésie basque contemporaine" (p. 133-139) est une affirmation très contestable. Pourquoi ne pas citer Gabriel Aresti le poète de la ville et du prolétariat qu'exécrait Jon Mirande ? Si on veut un panorama de la "production discursive" d'origine littéraire au $\mathrm{XX}^{\mathrm{e}}$ siècle, l'honnêteté intellectuelle et la rigueur épistémologique imposent pour le moins de mettre en parallèle les apports divers et contrastés de Jon Mirande et Gabriel Aresti, ou ceux de Lauaxeta (fusillé par les franquistes), Ricardo Arregi qui, malgré sa courte vie (1942-1969), est à l'origine d'une oeuvre pleine de promesses constituant aussi une "production discursive" renouvelée et moderne sur la "basquité".

La phrase par laquelle P.B. conclut cette troisième partie laisse perplexe: "Ce faisant, la basquité a été entraînée pour la première fois au centre d'une constellation conceptuelle et idéologique (..) au profit d'une pensée nauséabonde mais au destin heureusement sans éclat" (p. 141).

Certes Jon Mirande était un fasciste (tout le monde en convient), alors que pendant le même temps la Soule avait un maquis qui a constitué récemment le sujet d'une Pastorale souletine rédigée par Jean-Louis Davant. Mais Jon Mirande n'a eu aucun impact politique en Pays Basque. L'a-t-il même cherché ? A quoi donc P. B. veut-il faire allusion ? A Eugène Goyeneche et la polémique dont il a fait l'objet il y a quelques années ? Et que fait-il des Eskualtzale qui ont contribué activement- et au prix de leur sang parfois-, à la Résistance? Que fait-il du groupe de Jacques Légasse (celui-ci est mort au combat à Monte Cassino). P. B. ignore l'histoire de Jacques Légasse, du commandant Ordoki, des maquis et des groupes de combat d'Eusko Jaurlaritza (le général de Gaulle la connaissait), il ignore 
aussi le travail de Jean-Claude Larronde sur "le mouvement euskalerriste".

La quatrième partie sur "les nouveaux savoirs et la basquité" (p. 142-226) met l'accent sur la "production discursive" en matière d'anthropologie physique, d'ethnographie, de linguistique. Là aussi, quelques remarques s'imposent. "Les essais de normalisation de l'alphabet basque" renvoient, selon l'auteur, à Chaho. Et pour les temps antérieurs, il faut au moins mentionner Joannes Leizarraga $\left(\mathrm{XVI}^{\mathrm{e}}\right.$ siècle), Axular $\left(\mathrm{XVII}^{\mathrm{e}}\right.$ siècle). En ce qui concerne "l'envers racial négatif de la basquité" (p. 154), P. B. se contente d'un simple recensement des publications sur le "thème racial" mais sans aucune mise en perspective historique désormais possible avec les travaux de chercheurs comme $B$. Cursente et Véronique Beriac.

P.B. souligne qu'au XIX siècle "la basquité se construit à la faveur decf l'objectivation d'une somme de différences de même qu'elle s'incarne dans une carte" (p. 159). Mais le phénomène existe dans toute la France au XIX $X^{\mathrm{e}}$ siècle. C'est le thème de l'anthropologie physique, du colonialisme fondé sur la supériorité des populations blanches de l'Europe. De ce point de vue, il n'y a pas davantage de singularité basque que de singularité française. Ou, dans un autre sens, il y a une "invention" du Pays Basque tout comme il y a une "invention de la France" pour reprendre le titre d'un ouvrage d'anthropologie (15). Reprenons la "production discursive" de l'histoire de France ; elle est aussi fondée sur le mythe de la "nation française" et de la "Grande nation", avant et après 1789 , relayé par le chauvinisme des sans-culottes, l'idéologie colonisatrice de la III République et de ses appareils d'Etat sur lesquels P.B. a d'ailleurs écrit jadis.

Manex Goyhenetche

\section{Caro Baroja, Julio: Le mythe du caractère national : méditations à rebrousse-poil, traduit par Jean-Paul Cortoda[?], [titre original : El mito del carácter nacional. Meditaciones a contrapelo, 1970|, Ed. Dufourg-Tandrup, Presses Universitaires de Bordeaux, 2001, 104 p.}

Julio Caro Baroja n'est pas assez connu en français : on connaît surtout deux ouvrages fondamentaux comme Les sorcières et leur monde et Le Carnaval. Il faut donc saluer l'initiative qui voit publier un ouvrage dont le titre même est révélateur et dont tout l'intérêt est souligné dans l'introduction sous la plume de Bernard Traimond, du département d'anthropologie de l'Université Victor Segalen Bordeaux 2. Je formulerai cependant une remarque sur le caractère de nouveauté prêté à cette édition: en effet il n'est pas exact de dire que les deux ouvrages cités ci-dessus sont les seuls ouvrages de J. Caro Baroja publiés en français. Ce même Mythe du caractère national a bel et bien déjà été publié en 1975 à Lyon aux éditions Féderop, collection minorités nationales, dans la même traduction du même traducteur (appelé Cortada en 1975, Cortoda cn 2001). La qualité de présentation de l'édition de 2001 est parfois défaillante. L'introduction (elle est inédite quant à elle) souffre de quelques défauts de forme ; on trouvera par exemple une phrase identique à quelque chose près p. 8 en fin du deuxième paragraphe et p. 9 en fin du troisième paragraphe, et la cohérence du texte dans ces pages-là n'est pas assurée. La traduction elle-même méritait 